Case Report

\title{
Median nerve entrapment secondary to carpal osteophyte: A case report
}

\author{
Yunus Doğramacı, Aydıner Kalacı, Yurdal Serarslan*, Ahmet Nedim Yanat, Anıl Emir \\ Department of Neurosurgery, Medical Faculty, Mustafa Kemal University, Hatay, Turkey
}

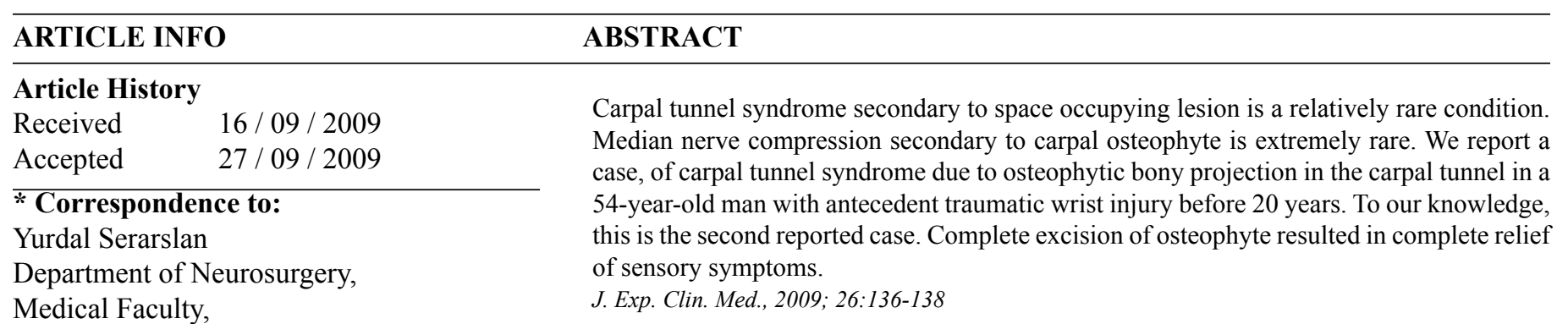

Mustafa Kemal University,

Hatay, Turkey

e-mail: yserarslan@yahoo.com

\author{
Key Words : \\ Carpal Tunnel Syndrome \\ Carpal Osteophyte \\ Median Nerve \\ Median Nerve Compression \\ Carpal Canal \\ Entrapment Neuropathy
}

(C) 2009 OMU All rights reserved

\section{Introduction}

Carpal Tunnel Syndrome (CTS) secondary to an osteophyte within the carpal canal is extremley rare. Space occupying lesions such as idiopathic calcified mass (Takada et al., 2000), heterotopic ossification (Wasman et al., 1997; Martinez et al., 2003), apatite mass (Sensui et al., 2003), hemangioma, synovial sarcoma, lipoma, ganglion and fibroma of tendon sheath have been identified as lesions that can cause CTS (Coessens et al., 1991; Weiss and Steichen, 1992; Nakamichi et al., 1994; Sensui et al., 2003). Median nerve entrapment due to a carpal osteophyte has been reported in only one previous case (Engel et al., 1978). We report a case of CTS due to a large carpal osteophyte secondary to extensive radiocarpal and intercarpal osteoartheritis.

\section{Case report}

A 54-year-old man complained of a 3-year history of numbness of his right thumb, index and middle fingers. There was a history of previous local trauma to the right wrist 20 -year ago that was managed by paramedical bonesetter. He had no history of previous medical illness that might have affected the hand. Movement of the cervical spine was normal, eliciting no neck pain or radiating pain. Physical examination of the right hand revealed swelling in the palmar and dorsal surface of the wrist joint with palpable hard mass in the palmar surface of the right wrist. There was no redness or local heat. Thenar muscle atrophy was identified. The range of motion of the wrist was no more than few degrees and restricted in all directions. The patient had hypoaesthesia in the median nerve distribution of the right hand. Both Tinel's sign and Phalen's test were positive. Laboratory studies including full blood count, erythrocyte sedimentation rate, and blood biochemistry were performed; all results including serum calcium, phosphate and alkaline phosphatase level were normal.

Radiographs of the right wrist revealed advanced osteoarthritic changes in the radiocarpal and intercarpal region (Fig. 1). There was an osteophyte in both volar and dorsal surface of the wrist joint and carpal region. Radiographs of the left wrist and hand revealed normal findings. Carpal tunnel view X-rays was not obtained as the elbow motion was severely restricted. Computed Tomography (CT) scan of the right wrist revealed advanced osteoarthritis of the intercarpal joints with a large osteophyte like lesion within the carpal canal at the level 


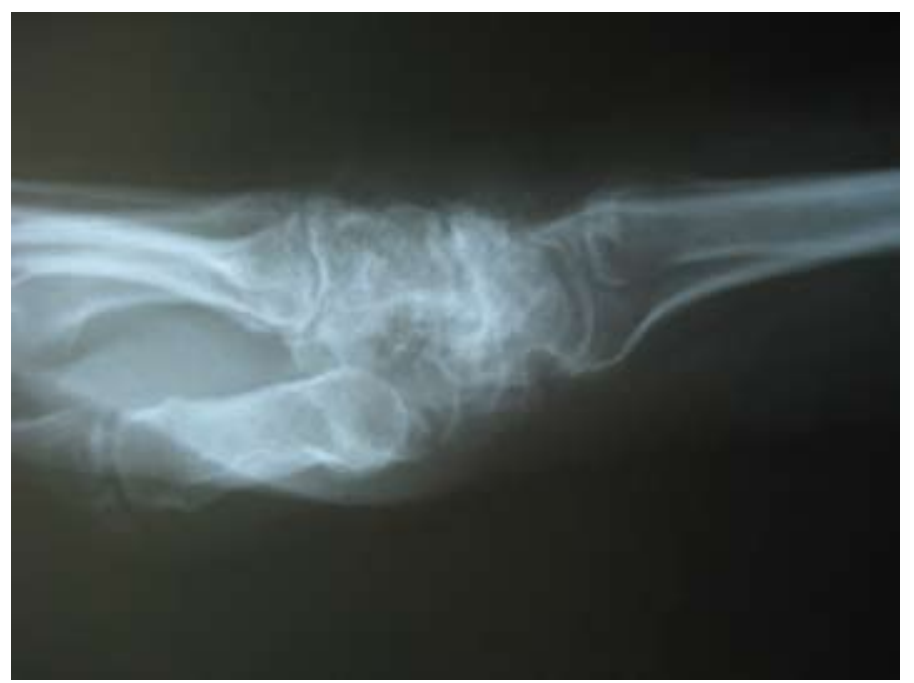

Fig. 1: Lateral X-ray of the right wrist showing calcification lying anterior to the carpus

of the proximal carpal row (Fig. 2). The carpal tunnel size reduced to less than $50 \%$. Motor nerve conduction studies of the right median nerve revealed a distal latency of 4.5 ms.

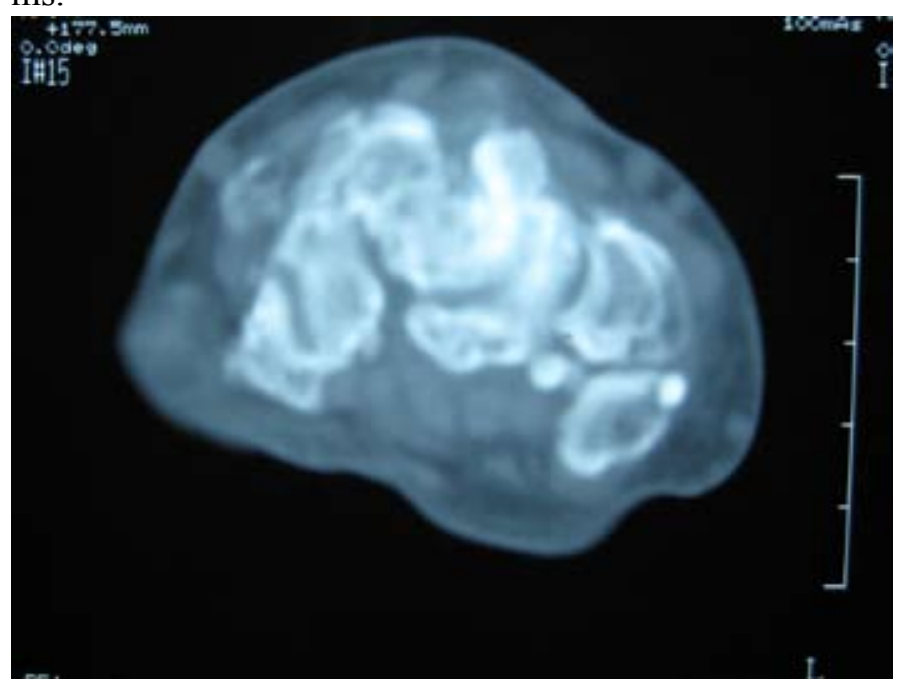

Fig. 2: CT scan showing volar osteophyte inside the carpal tunnel and features of advanced carpal arthroses.

Surgical exploration of the carpal tunnel was performed under general anesthesia. The transverse carpal ligament divided and the median nerve was found to be compressed between the transverse carpal ligament and the mass which measured $2.0 \times 2.0 \mathrm{~cm}$. It was found deep to the median nerve and flexor tendons and was attached to the scaphoid bone (Figs. 3a and b). Total resection of the mass was performed using an osteotome blade. Histologically, the mass was consistent with osteophyte. Numbness in the right hand disappeared in 1 month, and volume of the thenar muscle was partially restored at the 1-year follow up with no recurrence of CTS.

\section{Discussion:}

Suspicion of local pathology, especially a space-occupying lesion, is needed when the CTS is unilateral and the aetiology is not clear. In a study involving 20 patients with unilateral CTS, space-occupying lesions were found to be
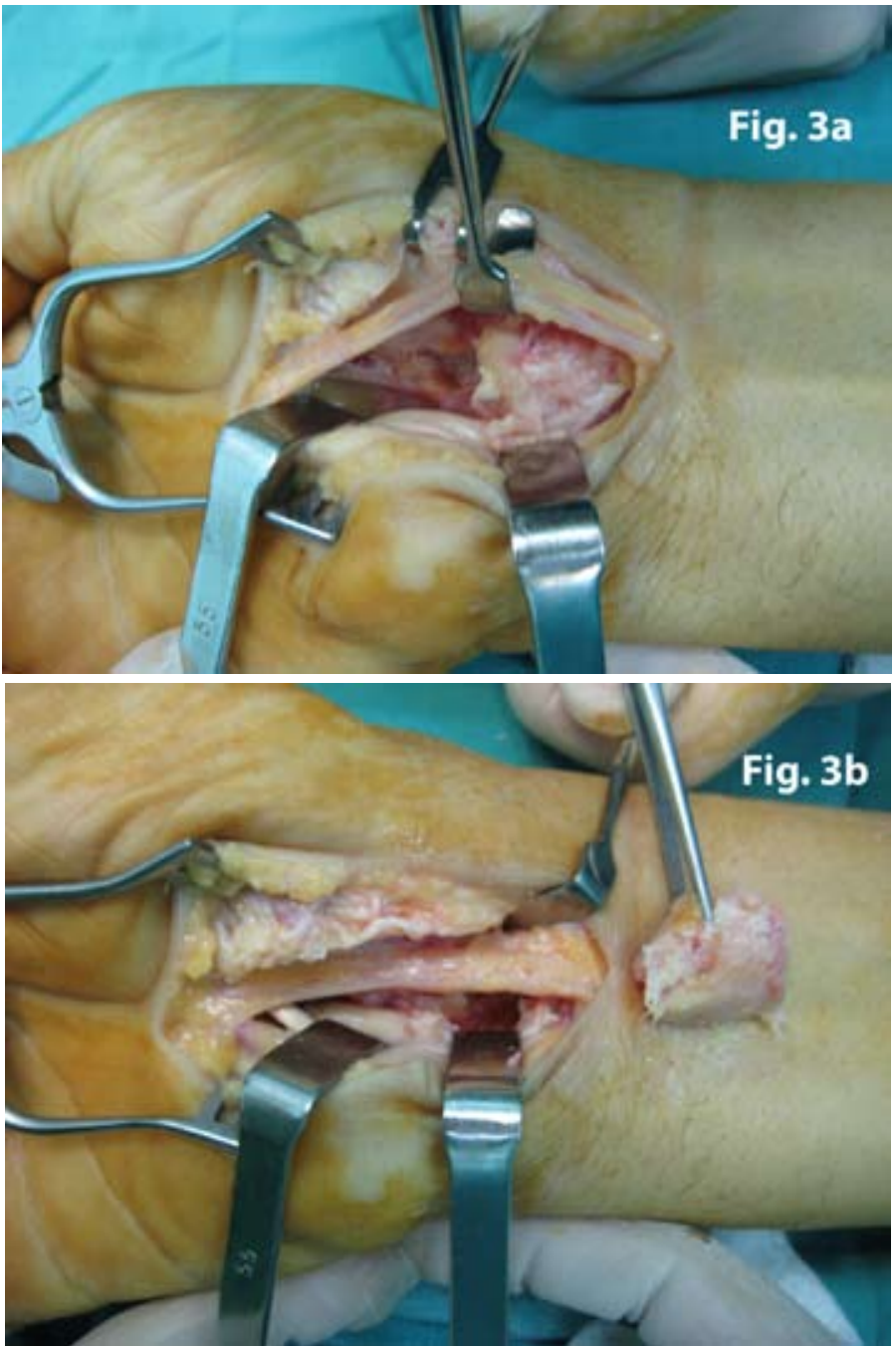

Fig. 3a and 3b: Intraoperative photograph showing the osteophyte (Fig. 3a), being removed from the carpal tunnel( Fig. 3b).

the cause of enterapment in seven patients (Nakamichi and Tachibana; 1993). Unilateral CTS resulting from carpal osteophytic lesions within the carpal canal is very rare and a review of the English language literature revealed only one reported case (Engel et al., 1978). Differential diagnosis of calcific ossifying lesions in the carpal canal includes extraskeletal chondroma (Cumming et al., 2005), heterotopic ossification, calcareous lesions, calcifying tendinitis or peritendinitis calcarea (Din et al., 2001), bone fragments from adjacent fractures and carpal osteophytes (Engel et al., 1978).

Wasman et al., (1997) reported an ossifying lesion arising within the median nerve, which was an intraneural ossification that histopathologically resembled to myositis ossificans (Martinez et al., 2003). Reported a case of CTS due to ideopathic heterotopic ossification in the carpal tunnel. As a complication of musculoskeletal trauma, the wrist is a highly unusual localization for heterotopic ossification. Its appearance on roentgenograms is that of a mature bone. Another reported calcific lesion has been a calcareous mass in the carpal tunnel. A calcareous mass may be caused by idiopathic calcification, gout, pseudogout,or tumoral calcinosis (Takada et al., 2000) described a case of CTS secondary to ideopathic calcareous mass within the carpal tunnel. In that case, the crystal type was calcium 
phosphate. They suggested that CTS could be caused by an isolated idiopathic calcium phosphate. In contrast, the crystal type has not been determined in CTS caused by an idiopathic calcareous mass (De, 1983). Laboratory tests may be of help in diagnosing secondary calcareous masses. In cases of gout, the elevated levels of uric acid in the blood are useful in suggesting this diagnosis, and the crystal type of the mass is uric acid (Ogilvie and Kay, 1988). In cases of pseudogout, calcium pyrophosphate is detected in the calcified material (Chiu et al., 1992). In tumoral calcinosis, calcareous lesions in the carpal canal associated with calcific periarthritis have been reported, and its crystal type was determined to be calcium phosphate by infrared absorption spectrometry (Weiber and Linell, 1987; Nakamichi et al., 1994).

Antero-posterior, lateral and carpal tunnel x-ray views are necessary for diagnosing calcific masses inside the carpal canal. CT is of great help in determining the size, location of the mass and for proper preoperative planning. Surgical resection of the mass with carpal tunnel decompression was curative in most of the reported cases.

In conclusion, space occupying lesions should be considered in the differential diagnosis of patients with unilateral CTS. CT is mandatory for diagnosing ossified masses in the carpal canal when the carpal tunnel view $\mathrm{X}$-rays are not helpful.

\section{REFERENCES}

Chiu, K.Y., Ng, W.F., Wong, W.B., Choi, C.H., Chow, S.P., 1992. Acute carpal tunnel syndrome caused by pseudogout. J. Hand Surg. 17, 299-302.

Coessens, B., De Mey, A., Lacotte, B., Vandenbroeck, D., 1991. Carpal tunnel syndrome due to an haemangioma of the median nerve in a 12-year-old child. Ann. Chir. Main. Memb. Super. 10, 255-257.

Cumming, D., Massraf, A., Jones, J.W., 2005. Extraskeletal chondroma as a cause of carpal tunnel syndrome. Hand Surg. 10, 327-330.

De, S.D, 1983. Carpal tunnel syndrome due to a calcareous mass in the carpal tunnel. Singapore Med. J. 24, $175-177$.

Din, R., Giannikas, K., El-Hadidi, M., 2001. Acute calcifying tendonitis-an unusual cause of carpal tunnel syndrome. Eur. J. Emerg. Med. 8, 65-66.

Engel, J., Zinneman, H., Tsur, H., Farin, I., 1978. Carpal tunnel syndrome due to carpal osteophyte. Hand 10, $283-284$.

Martinez, A., Peguero, A., Canales, V., Herrera, A., 2003. Carpal tunnel syndrome due to heterotopic ossification. J. Orthop. Sci. 8, 596-598.

Nakamichi, K., Tachibana, S., 1993. Unilateral carpal tunnel syndrome and space-occupying lesions. J. Hand Surg. 18, 748-749.

Nakamichi, K., Tachibana, S., Tamai, K., 1994. Carpal tunnel syndrome caused by a mass of calcium phosphate. J. Hand Surg. 19, $111-113$.

Ogilvie, C., Kay, N.R., 1988. Fulminating carpal tunnel syndrome due to gout. J. Hand Surg. [Br]. 13, $42-43$.

Sensui, K., Saitoh, S., Kametani, K., Makino, K., Ohira, M., Kimura, T., Cheng, G.A., Hata, Y., 2003. Property analysis of ectopic calcification in the carpal tunnel identification of apatite crystals: a case report. Arch. Orthop. Trauma Surg. 123, $442-445$.

Takada, T., Fujioka, H., Mizuno, K., 2000. Carpal tunnel syndrome caused by an idiopathic calcified mass. Arch. Orthop. Trauma. Surg. 120, 226-227.

Wasman, J.K., Willis, J., Makley, J., Abdul-Karim, F.W., 1997. Myositis ossificans-like lesion of nerve. Histopathology. 30 , 75-78.

Weiber, H., Linell, F., 1987. Tumoral calcinosis causing acute carpal tunnel syndrome. Case report. Scand. J. Plast. Reconstr. Surg. Hand Surg. 21, 229-230.

Weiss, A.P., Steichen, J.B., 1992. Synovial sarcoma causing carpal tunnel syndrome. J. Hand. Surg. 17, $1024-1025$. 